\title{
The Role of DSM During the COVID 19 Disease Crisis: Management with CPAP and Oral Appliances
}

\author{
Jean-François Masse, DMD, MSc, FACD, Diplomate, ABDSM
}

Editor-in-Chief Journal of Dental Sleep Medicine Universite Laval, Quebec City, Quebec, Canada

It is obvious that over the last century, dentists have learned to develop highly effective methods to control the transmission of infectious disease such as advanced sterilization techniques, use of personal protective equipment and surface disinfection, among others. Now, we have another major challenge - how can we help our patients with the COVID-19 disease induced by the novel virus, the SARS Cov-2? It seems that $95 \%$ to $99 \%$ of those infected will recover. How well they will recover is not yet defined; long-term impact is still unknown as some viruses may have latent effect on living species.

The topic is particularly timely and interesting. Dr. Gary Kobinger, the scientist who developed the first effective vaccine against the Ebola virus, argued that it was only a matter of time before another pandemic developed. ${ }^{1}$ Unfortunately, it seems we will have to get used to such global events and adjust our practices with resilience. These viral crises may be periodic; the 2009 H1N1 pandemic caused up to $575,400^{2}$ deaths, the 2013 Ebola over $11,000^{3}$ and the current one is escalating.

Keeping all this in mind, how should sleep dentists be proactive?

Determining how to best prevent transmission is now the key to reducing the virus propagation. As medical or dental sleep clinicians, we need to guide and manage sleep patients using CPAP and/or oral appliance, both with and without the COVID-19 disease. We have not yet developed a proactive strategy for those with recurrent high-risk viral attacks. We are building knowledge that will drive us toward evidence-based data and future recommendations. At this time, we have to be cautious and act in accordance with public health advice and guidelines.

\section{CPAP issues:}

The AASM recently released recommendations regarding the use of CPAP at home for patients who have been diagnosed with or are suspected to have COVID19. These prudent recommendations are based on a case-by-case benefit-risk assessment, the major concern being directed toward people living in the same home as the CPAP users.

According to current CDC guidelines, CPAP users with respiratory infections should ideally be isolated from other people. To prevent airdrop diffusion in the patient room, confinement is the best solution. The isolation of a coronavirus-OSA patient, non-intubated and using a CPAP, is relatively simple if the person stays at home. However, it is a major challenge for CPAP users in a hospital or in retirement home. Family and staff entering and leaving the room, going home after work or visiting other hospitalized patients is also a risk vector of the spread of infection (not just with COVID-19, but any virus).

As far as COVID-19 is concerned, we know that the new coronavirus is stable for hours if not days on surfaces (up to three days on plastic and stainless steel). ${ }^{5}$ We also know that airborne particles are being dispersed when a patient uses CPAP. It is also thought that the dispersion is limited provided there is good mask interface fitting. ${ }^{6}$ This research, despite being well done, has been performed on models. Keep in mind that a living, moving sleeping patient could provide us with different results.

Additionally, how to 'effectively' disinfect CPAP device, hoses and masks is not clear for many users and caregivers. It may not be a top priority in the actual intense period dealing with many issues to prevent SARS CoV-2 spread and how this knowledge is applied in hospital or retirement home is unclear.

U.S. hospitals are facing widespread shortages of vital medical equipment, including ventilators and personal protective equipment. ${ }^{7}$ During the last weekend of March 2020, Dr. Anthony S Fauci, the director of the National Institute of Allergy and Infectious Diseases in USA, sent a clear and strong message about the magnitude of the viral spread and risks.

The latest FDA recommendations mentions use of CPAP if there are no ventilators available. ${ }^{8}$ At the same time, they added a warning about the danger of virus propagation with CPAP. It is clear that lack of evidence propagates confusion! This idea obviously needs to be scientifically validated, but as an infectious disease specialist said the other day about adapting to the everchanging measures regarding the pandemic crisis: it is like we are building the plane as we are flying it... Some 
urgent actions will have to be take in the ongoing crisis; these have to be based on logic, best science available and not on fear or some 'obscure' interest.

\section{Oral Appliance issues:}

Is it tempting to propose oral appliances as a replacement for CPAPs when indicated for nonintubated patient? While a difficult issue to clarify, we have to keep in mind that many OSA patients are also mouth breathers, and thus, respiratory aerosol can still be a problem. To do no harm, caution may be the wisest route to take for now.

Combination treatment, i.e. CPAP with an oral appliance, to prevent mask leak may be seen by some as an alternative. Again, it is premature to confirm if this route is safe. We know from El-Solh pilot study ${ }^{9}$ combining CPAP with OA not only significantly reduced the CPAP pressure (thus potentially reducing the amount of aerosols) but also increased patient comfort with the CPAP. However, we have to wait for more solid evidence before moving into that direction.

Which brings me to another related topic - the apparent absence of validated protocols for the disinfection of oral appliances. Considering the situation we are going through right now and that OSA patients can present with more comorbidities than regular denture patients or orthodontic appliance patients, it is time to start doing analyses of past actions and proactively look at what can we do to minimize the risk of infections related to the wear of oral appliances. A review of the literature shows that so far, no case of disease associated with the wear of a dental sleep appliance has been reported. A rapid survey of the literature also shows us the absence of solid studies related to the disinfection of oral appliances for OSA. It is critical to study which method of disinfection is both effective against SARS CoV-2 and yet will not alter the material properties of the OA. Good ideas have to be tested.

CPAP companies, like ResMed and Philips ${ }^{10,11}$ have their own 'validated' protocols for the disinfection of CPAP machines. In light of this, should dental manufacturers create protocols suited for their own appliances? Should dental labs institute rules regarding disinfection (at the dental office and in the lab) when we adjust oral appliances and send cases back to fabricant for repair? Should we modify our informed consent forms to include a section on disinfection, not only to protect our patients and family, but also to protect ourselves when patients come for their visits?

So many unanswered questions that will need to be addressed in the near future. If you think this is a bit of a stretch, this is precisely what I thought when as a dental student, I had to switch from treating patients from bare hands to gloves. Nowadays, a dentist not wearing gloves would be reported to their dental board.
We know that qualified DSM dentists can help physicians. Now more than ever, physicians are overburdened and are going to need to rely on those qualified dentists to help manage OSA. Dentists should be having conversations with their physicians on how they can help - be that screening, triaging patients, or changing protocols to expedite follow-up care. We should also be speaking with primary care physicians who will be worried about comorbidities associated with sleep apnea more than ever. In this moment, HIPPA regulations are being relaxed to allow providers to a wide variety of platforms to video chat and virtually communicate with patients ${ }^{12}$; dentists can utilize telehealth to keep patients engaged and continue treatment of sleep apnea.

Times are changing and this pandemic crisis will have a dramatic impact on the way we practice and the way we live. We have to "do no harm" and we need to be proactive, base our action on evidence and, as usual, be resilient. Adaptation is the key of species survival and dentists are among the champions in such processes. Are we ready for the next steps?

We can only hope it will be for the best.

\section{CITATION}

Masse, JF. The Role of DSM During the COVID 19

Disease Crisis: Management with CPAP and Oral

Appliances. J Dent Sleep Med. 2020;7(2)

\section{REFERENCES}

1. Se préparer pour un virus plus grave. https://www.lapresse.ca/covid-19/202003/17/01-5265168-sepreparer-pour-un-virus-plus-grave.php. Accessed March 30, 2020

2. 2009 H1N1 Pandemic (H1N1pdm09 virus). CDC https://www.cdc.gov/flu/pandemic-resources/2009-h1n1pandemic.html. Accessed March 30, 2020.

3. 2014-2016 Ebola Outbreak in West Africa. CDC https://www.cdc.gov/vhf/ebola/history/2014-2016outbreak/index.html. Accessed March 30, 2020.

4. COVID-19: FAQs for Sleep Medicine Clinicians and Sleep Facilities. AASM. https://aasm.org/covid-19-resources/covid-19faq. Accessed March 30, 2020.

5. Study suggests new coronavirus may remain on surfaces for days National Institutes of Health (NIH). https://www.nih.gov/newsevents/nih-research-matters/study-suggests-new-coronavirusmay-remain-surfaces-days. Accessed March 30, 2020.

6. Hui DS, Chow BK, Lo T, et al. Exhaled air dispersion during highflow nasal cannula therapy versus CPAP via different masks. Eur Respir J. 2019;53(4). doi:10.1183/13993003.02339-2018

7. Ranney ML, Griffeth V, Jha AK. Critical Supply Shortages - The Need for Ventilators and Personal Protective Equipment during the Covid-19 Pandemic. N Engl J Med. March 2020:NEJMp2006141. doi:10.1056/NEJMp2006141

8. Ventilator Supply Mitigation Strategies: Letter to Health Care Providers. FDA. https://www.fda.gov/medical-devices/letters- 
health-care-providers/ventilator-supply-mitigation-strategiesletter-health-care-providers. Accessed March 30, 2020.

9. El-Solh AA, Moitheennazima B, Akinnusi ME, Churder PM, Lafornara AM. Combined oral appliance and positive airway pressure therapy for obstructive sleep apnea: a pilot study. Sleep Breath. 2011;15(2):203-208. doi:10.1007/s11325-010-0437-1

10. Cleaning \& disinfection support. ResMed. https://www.resmed.com/epn/en/healthcare-

professional/support/cleaning---disinfection-support.html. Accessed March 30, 2020.

11. Keeping it clean: CPAP hygiene. Philips. https://www.usa.philips.com/c-e/hs/better-sleep-breathingblog/better-sleep/keeping-it-clean-cpap.html. Accessed March 30, 2020.

12. Notification of Enforcement Discretion for Telehealth. HHS.gov. https://www.hhs.gov/hipaa/for-professionals/specialtopics/emergency-preparedness/notification-enforcementdiscretion-telehealth/index.html. Accessed April 1, 2020

\section{SUBMISSION AND CORRESPONDENCE INFORMATION}

\section{Submitted in final revised form April 2, 2020.}

Address correspondence to: Jean-François Masse, DDS, MSc, FACD, D.ABDSM, Professor, Universite Laval, 2780 Masson \#200, Quebec City, QC, G1P 1J6, Canada; Tel: 418871-1447; Fax: 418-871-4983; Email: jean-francois.masse@fmd.ulaval.ca 